\title{
Prediction of Market Weight in Caribro-Dhanraja Broilers with Different Plumage Colour Using Growth Traits
}

\author{
T.K. Patbandha ${ }^{1 *}$, D.D. Garg ${ }^{1}$, D.G. Vaghamashi ${ }^{1}$, S. Marandi ${ }^{1}$, K. Ravikala ${ }^{1}$, \\ S.S. Patil ${ }^{2}$ and S.K. Dash ${ }^{3}$ \\ ${ }^{1}$ College of Veterinary Science and A.H., Junagadh Agricultural University, \\ Junagadh-362001, Gujarat, India \\ ${ }^{2}$ College of Veterinary Science and A.H., SDAU, Sardarkrushi nagar -385506, Gujarat, India \\ ${ }^{3}$ College of Veterinary Science, GADVASU, Ludhiana-141004, Punjab, India \\ *Corresponding author
}

\begin{abstract}
A B S T R A C T
Present study predicted $6^{\text {th }}$ week body weight of Caribro-Dhanraja broilers $(n=75)$ with different plumage colour through backward elimination regression from $2^{\text {nd }}$ week growth traits (weight, weight gain, chick length, weight: length ${ }^{2}$, shank length, toe length, breast length, breast girth, thigh length and wing length). Day old chicks were grouped into yellow, black and stripped colour groups based on plumage colour (25 chicks in each

\section{Keywords}

Caribro-Dhanraja, Coloured broiler, Growth traits

\section{Article Info}

Accepted: 08 August 2018 Available Online: 10 September 2018 group) and traits were measured biweekly upto $6^{\text {th }}$ week. Plumage colour significantly influenced shank, toe and thigh length, and breast girth at $2^{\text {nd }}$ week; whereas, wing length at $4^{\text {th }}$ week $(\mathrm{P} \leq 0.05)$. In yellow plumage group, weight, daily gain, weight: length ${ }^{2}$, shank, breast and thigh length at $2^{\text {nd }}$ week were positively associated with $6^{\text {th }}$ week body weight $(\mathrm{P} \leq 0.05)$. In black plumage group, weight, daily gain and weight: length ${ }^{2}$ of $2^{\text {nd }}$ week were positively association with $6^{\text {th }}$ week body weight, but thigh length was negatively associated $(\mathrm{P} \leq 0.05)$. Weight, daily gain and weight: length ${ }^{2}$ at $2^{\text {nd }}$ week were positively associated with $6^{\text {th }}$ week body weight in stripped plumage group $(\mathrm{P} \leq 0.05)$. Backward elimination regression model for prediction of $6^{\text {th }}$ week body weight from $2^{\text {nd }}$ week variables revealed higher $\mathrm{R}^{2}$ value $(59.6 \%)$ in black plumage (predictor variables were weight, breast girth and thigh length). However, $\mathrm{R}^{2}$ value was $36 \%$ for stripped colour (predictor variables were weight and breast girth), $25.3 \%$ for yellow plumage (predictor variable was wing length) and $41 \%$ for pooled data (predictor variables were growth and breast girth). The results indicated lower accuracy for prediction of $6^{\text {th }}$ week body weight based on variables from $2^{\text {nd }}$ week.
\end{abstract}

\section{Introduction}

Poultry meat is well accepted by people of all religions throughout the world. In the present scenario, poultry meat production of the world is dominated by white-feathered broilers because of their fast growth rate along with better feed efficiency (Yang and Jiang, 2005). Slow growing coloured broilers have great consumers' demand owing to better quality meat in terms of appearance, less fat and taste as well as better performance in tropical 
climate than fast growing white feathered broilers (Yang and Jiang, 2005, Padhi, 2016). Further, coloured chickens fetch more price than the white feathered fast growing broilers (Bett et al., 2011). Identification of broilers with better growth potential at an early age is most essential for better economic return as inferior birds can be culled early.

Chick quality traits, particularly weight of chick has been commonly used to assess growth performance at later age, but such effect is inconsistent and diminished well in advance before the market age (Jiang et al., 2007; Molenaar et al., 2008; Patbandha et al., 2017). Moreover, others reported positive effect of body linear traits like chick body length on growth performance up to market age (Molenaar et al., 2008; Mukhtar et al., 2013; Patbandha et al., 2017). Association of shank and toe length at early age with future growth performance was observed to be inconsistent up to market age in different studies (Wolanski et al., 2006; Willemsen et al., 2008; Patbandha et al., 2017). However, information regarding association of traits like daily gain, body mass index (weight: length ${ }^{2}$ ), toe length, breast length, breast girth, thigh length and wing length with market weight is scanty.

Further, significant effect of plumage colour on growth traits of chicks within a particular strain has been well established (Azahan, 1994; Rizzi et al., 2013; Rizzi, 2017). Additionally, variable selection for regression model using backward elimination (where initially model includes all variables and later deleted one by one based on $F$-statistics) has been reported to be best among the all stepwise procedures (Sauerbrei et al., 2007). Hence, the present study was designed to predict the market weight of Caribro-Dhanraja broiler chicken with different plumage colour using backward elimination regression model of different early growth traits.

\section{Materials and Methods}

\section{Experimental birds and general management}

The experiment was conducted on CaribroDhanraja coloured broiler strain during June to August, 2017 at Instructional Livestock Farm Complex, Junagadh Agricultural University, Junagadh (Gujarat). Junagadh is located with a latitude of $21^{\circ} 31^{\prime} \mathrm{N}$ and an altitude of $70^{\circ} 36^{\prime}$ E under South Saurashtra Agroclimatic zone. Day old, physically healthy straight run chicks ( $n=75$ with 25 chicks from each yellow, black and stripped colour) were included for this study. The chicks were numbered individually using wing band at the start of the experiment. The chicks were reared under deep litter system of housing with uniform managemental conditions as per the farm standards. Lighting facilities were switched on before few hours of arrival of chicks to provide the required temperature for brooding and provided continuously during the study period for 24 hours. Experimental birds were vaccinated for poultry diseases (Marek's disease and New castle disease) as per the guidelines for commercial broilers. Birds were provided with commercial starter mash $(23 \%$ $\mathrm{CP}$ and $2800 \mathrm{Kcal} \mathrm{ME}$ per $\mathrm{kg}$ ) and finisher mash $(20 \% \mathrm{CP}$ and $2900 \mathrm{Kcal} \mathrm{ME}$ per $\mathrm{kg}$ diets) with ad libitum access to feed and water.

\section{Measurement of growth traits}

Growth traits of individual birds such as body weight, length of chick, shank, toe, breast, thigh, wing and girth of breast were measured at 2 weeks interval up to 6 weeks. All the traits were measured during morning before feeding and watering. Daily gain and body mass index (weight: length ${ }^{2}$ ) were calculated. Chick length was defined as length from tip of beak to length of longest toe excluding its nail and measured by keeping the chick on a 
platform on its ventral aspect in such a position by extending neck and toe to their maximum extent gently. Length from hock joint to bottom of foot pad was considered as shank length; whereas, length of longest toe upto insertion of nail was defined as toe length. Breast length and girth were defined as length between both vertices of the sternum and circumference of the breast region, respectively. Wing length was measured from scapula joints to the last digit of the wing and thigh length was measured from shinbonefemur joint to shinbone-tarsus joint.

\section{Statistical analysis}

The data of growth traits were collected and presented as mean with standard error estimates. The growth traits of broilers among the different plumage groups were compared by one-way analysis of variance and Duncan's multiple range tests was used as post hoc test to compare the pair wise mean differences. Correlation of different growth traits at $2^{\text {nd }}, 4^{\text {th }}$ and $6^{\text {th }}$ week with body weight at $6^{\text {th }}$ week was estimated by Pearson correlation. Backward elimination regression model (Chatterjee et al., 2000) was used for prediction of $6^{\text {th }}$ week body weight from variables recorded at $2^{\text {nd }}$ week of age. The variables were removed one by one from the model when probability of ' $F$ ' $>0.1$. The statistical analysis was carried out using SPSS software package (Version 16.0, USA) and considered as significant when ' $P$ ' $\leq 0.05$.

\section{Results and Discussion}

\section{Growth traits}

Body weight and conformation traits of coloured broiler chicken are presented in table 1. The body weight of coloured broilers was observed to be $1717.75 \mathrm{~g}$ at $6^{\text {th }}$ week with feed conversion ratio (0-6 weeks) of 2.16. Plumage colour did not affect body weight, daily body gain, chick length, weight: length ${ }^{2}$ and breast length of broilers during the study period. There was significant effect of plumage colour on shank length $(\mathrm{P}<0.05)$, toe length $(\mathrm{P}<0.001)$, breast length $(\mathrm{P}<0.05)$ and thigh length $(\mathrm{P}<0.05)$ at $2^{\text {nd }}$ week of age. However, at $4^{\text {th }}$ week the wing length was significantly affected by the plumage colour in coloured broilers $(\mathrm{P}<0.01)$.

The live weight of CARIBRO-Dhanraja observed in this study is comparatively higher than the previous studies (Singh, 2008; Bhonsle, 2009; Tomar et al., 2011; Anonymous, 2003, 2017), who reported $6^{\text {th }}$ week body weight about 769-1580g. This variation might be attributed to different managerial practices as well as climatic alteration in different regions. Further, comparatively higher value of body confirmatory traits were observed in this study than the others (Singh, 2008; Singh and Jilani, 2008; Bhonsle, 2009) may be the reason of higher live weight of broilers as these parameters are positively associated with live weight.

Plumage colour did not influence body weight in Caribro-Dhanraja chickens which is in consonance with Azahan (1994), who observed similar weight in red and black-red native or Kampung chickens of Malaysia. In case of Padovana chickens of Italy, previous studies reported significant effect of plumage colour on daily growth (Rizzi et al., 2013; Rizzi, 2017), such effect was not observed in Caribro-Dhanraja. The Padovana chicken has slow growth rate $(<20 \mathrm{~g} / \mathrm{day}$, Rizzi et al., 2013); whereas, Caribro-Dhanraja has better growth rate of $23.43-49.24 \mathrm{~g} / \mathrm{d}$, might be attributed to variation of results between the studies. Additionally, Rizzi (2017) observed the effect of plumage colour on daily growth rate up to the maturity age in Padovana chickens and beyond that such effect was nullified. 
Table.1 Body weight and conformation traits of coloured broiler chicken

\begin{tabular}{|c|c|c|c|c|}
\hline Weeks & Yellow $(n=25)$ & Black $(n=25)$ & Stripped $(n=25)$ & Overall $(n=75)$ \\
\hline \multicolumn{5}{|c|}{ Body weight (g) } \\
\hline $2^{\text {nd }}$ week & $368.84 \pm 6.53$ & $375.32 \pm 8.57$ & $372.60 \pm 6.31$ & $372.25 \pm 4.12$ \\
\hline $4^{\text {th }}$ week & $1021.76 \pm 21.93$ & $1043.40 \pm 22.82$ & $1020.04 \pm 17.87$ & $1028.40 \pm 12.01$ \\
\hline $6^{\text {th }}$ week & $1716.00 \pm 45.83$ & $1728.44 \pm 43.41$ & $1708.80 \pm 38.52$ & $1717.75 \pm 24.33$ \\
\hline \multicolumn{5}{|c|}{ Daily gain (g/day) } \\
\hline 0-2 week & $23.18 \pm 0.44$ & $23.69 \pm 0.59$ & $23.41 \pm 0.44$ & $23.43 \pm 0.28$ \\
\hline 2-4 week & $46.64 \pm 1.27$ & $47.72 \pm 1.19$ & $46.25 \pm 0.99$ & $46.87 \pm 0.66$ \\
\hline 4-6 week & $49.59 \pm 1.84$ & $48.93 \pm 1.80$ & $49.20 \pm 1.67$ & $49.24 \pm 1.01$ \\
\hline \multicolumn{5}{|c|}{ Chick length $(\mathrm{cm})$} \\
\hline $2^{\text {nd }}$ week & $33.60 \pm 0.21$ & $33.25 \pm 0.27$ & $33.70 \pm 0.20$ & $33.52 \pm 0.13$ \\
\hline $4^{\text {th }}$ week & $47.36 \pm 0.32$ & $48.02 \pm 0.26$ & $48.07 \pm 0.29$ & $47.82 \pm 0.17$ \\
\hline $6^{\text {th }}$ week & $57.92 \pm 0.46$ & $58.22 \pm 0.37$ & $58.76 \pm 0.37$ & $58.30 \pm 0.23$ \\
\hline \multicolumn{5}{|c|}{ Weight: Length ${ }^{2}\left(\mathrm{~g} / \mathrm{cm}^{2}\right)$} \\
\hline $2^{\text {nd }}$ week & $0.326 \pm 0.004$ & $0.340 \pm 0.008$ & $0.328 \pm 0.006$ & $0.331 \pm 0.004$ \\
\hline $4^{\text {th }}$ week & $0.455 \pm 0.007$ & $0.451 \pm 0.006$ & $0.441 \pm 0.005$ & $0.449 \pm 0.004$ \\
\hline $6^{\text {th }}$ week & $0.509 \pm 0.008$ & $0.508 \pm 0.008$ & $0.494 \pm 0.007$ & $0.504 \pm 0.005$ \\
\hline \multicolumn{5}{|c|}{ Shank length $(\mathrm{cm})$} \\
\hline $2^{\text {nd }}$ week & $5.65 \pm 0.07^{\mathrm{ab}}$ & $5.50 \pm 0.09^{\mathrm{a}}$ & $5.74 \pm 0.05^{\mathrm{b}}$ & $5.63 \pm 0.04 *$ \\
\hline $4^{\text {th }}$ week & $8.04 \pm 0.07$ & $7.96 \pm 0.08$ & $8.10 \pm 0.06$ & $8.03 \pm 0.04$ \\
\hline $6^{\text {th }}$ week & $9.68 \pm 9.68$ & $9.80 \pm 9.80$ & $9.84 \pm 9.84$ & $9.77 \pm 9.77$ \\
\hline \multicolumn{5}{|c|}{ Toe length $(\mathrm{cm})$} \\
\hline $2^{\text {nd }}$ week & $4.24 \pm 0.05^{\mathrm{a}}$ & $4.18 \pm 0.03^{\mathrm{a}}$ & $4.54 \pm 0.07^{b}$ & $4.32 \pm 0.03 * * *$ \\
\hline $4^{\text {th }}$ week & $5.76 \pm 0.05$ & $5.84 \pm 0.08$ & $5.72 \pm 0.05$ & $5.77 \pm 0.03$ \\
\hline $6^{\text {th }}$ week & $6.80 \pm 0.08$ & $6.83 \pm 0.07$ & $6.72 \pm 0.06$ & $6.78 \pm 0.04$ \\
\hline \multicolumn{5}{|c|}{ Breast length $(\mathrm{cm})$} \\
\hline $2^{\text {nd }}$ week & $7.54 \pm 0.08$ & $7.41 \pm 0.08$ & $7.50 \pm 0.07$ & $7.48 \pm 0.05$ \\
\hline $4^{\text {th }}$ week & $11.12 \pm 0.13$ & $11.16 \pm 0.11$ & $11.09 \pm 0.10$ & $11.12 \pm 0.06$ \\
\hline $6^{\text {th }}$ week & $13.32 \pm 0.15$ & $13.48 \pm 0.13$ & $13.50 \pm 0.09$ & $13.43 \pm 0.07$ \\
\hline \multicolumn{5}{|c|}{ Breast girth $(\mathrm{cm})$} \\
\hline $2^{\text {nd }}$ week & $17.27 \pm 0.10^{\mathrm{a}}$ & $16.81 \pm 0.15^{b}$ & $17.17 \pm 0.13^{\mathrm{ab}}$ & $17.08 \pm 0.08 *$ \\
\hline $4^{\text {th }}$ week & $24.17 \pm 0.19$ & $24.64 \pm 0.21$ & $24.16 \pm 0.16$ & $24.32 \pm 0.11$ \\
\hline $6^{\text {th }}$ week & $29.24 \pm 0.40$ & $29.69 \pm 0.39$ & $29.02 \pm 0.25$ & $29.32 \pm 0.21$ \\
\hline \multicolumn{5}{|c|}{ Thigh length $(\mathrm{cm})$} \\
\hline $2^{\text {nd }}$ week & $7.51 \pm 0.07^{\mathrm{a}}$ & $7.08 \pm 0.08^{b}$ & $7.61 \pm 0.07^{\mathrm{a}}$ & $7.40 \pm 0.05^{*}$ \\
\hline $4^{\text {th }}$ week & $10.48 \pm 0.08$ & $10.33 \pm 0.11$ & $10.56 \pm 0.06$ & $10.45 \pm 0.05$ \\
\hline $6^{\text {th }}$ week & $12.75 \pm 0.13$ & $12.89 \pm 0.13$ & $12.88 \pm 0.10$ & $12.84 \pm 0.07$ \\
\hline \multicolumn{5}{|c|}{ Wing length $(\mathrm{cm})$} \\
\hline 2nd week & $11.21 \pm 0.09$ & $11.29 \pm 0.28$ & $11.02 \pm 0.08$ & $11.17 \pm 0.10$ \\
\hline 4th week & $17.74 \pm 0.15^{\mathrm{a}}$ & $17.10 \pm 0.20^{b}$ & $17.85 \pm 0.12^{\mathrm{a}}$ & $17.57 \pm 0.10 * *$ \\
\hline $6^{\text {th }}$ week & $22.13 \pm 0.20$ & $21.93 \pm 0.20$ & $22.13 \pm 0.17$ & $22.06 \pm 0.11$ \\
\hline
\end{tabular}


Table. 2 Correlation of $2^{\text {nd }} 4^{\text {th }}$ and $6^{\text {th }}$ week body weight and chick conformation with weight at $6^{\text {th }}$ week in coloured broiler chicken

\begin{tabular}{|c|c|c|c|c|c|c|c|c|c|c|}
\hline Weeks & $\begin{array}{c}\text { Body } \\
\text { weight }\end{array}$ & $\begin{array}{l}\text { Daily } \\
\text { gain }\end{array}$ & $\begin{array}{l}\text { Chick } \\
\text { length }\end{array}$ & $\begin{array}{l}\text { Weight: } \\
\text { Length }^{2}\end{array}$ & $\begin{array}{l}\text { Shank } \\
\text { length }\end{array}$ & $\begin{array}{c}\text { Toe } \\
\text { length }\end{array}$ & $\begin{array}{l}\text { Breast } \\
\text { length }\end{array}$ & $\begin{array}{l}\text { Breast } \\
\text { girth }\end{array}$ & $\begin{array}{l}\text { Thigh } \\
\text { length }\end{array}$ & $\begin{array}{l}\text { Wing } \\
\text { length }\end{array}$ \\
\hline $\mathrm{X}_{2} \cdot \mathrm{WT}_{6}$ & $\begin{array}{c}0.593 \\
(0.002)\end{array}$ & $\begin{array}{c}0.606 \\
(0.001)\end{array}$ & $\begin{array}{c}0.241 \\
(0.246)\end{array}$ & $\begin{array}{c}0.586 \\
(0.002)\end{array}$ & $\begin{array}{c}0.419 \\
(0.037)\end{array}$ & $\begin{array}{c}0.289 \\
(0.161)\end{array}$ & $\begin{array}{c}0.498 \\
(0.011)\end{array}$ & $\begin{array}{c}0.340 \\
(0.096)\end{array}$ & $\begin{array}{c}0.399 \\
(0.048)\end{array}$ & $\begin{array}{c}0.261 \\
(0.208)\end{array}$ \\
\hline $\mathrm{X}_{4} \cdot \mathrm{WT}_{6}$ & $\begin{array}{c}0.954 \\
(0.000)\end{array}$ & $\begin{array}{c}0.957 \\
(0.000)\end{array}$ & $\begin{array}{c}0.593 \\
(0.002)\end{array}$ & $\begin{array}{c}0.785 \\
(0.000)\end{array}$ & $\begin{array}{c}0.833 \\
(0.000)\end{array}$ & $\begin{array}{c}0.523 \\
(0.007)\end{array}$ & $\begin{array}{c}0.762 \\
(0.000)\end{array}$ & $\begin{array}{c}0.703 \\
(0.000)\end{array}$ & $\begin{array}{c}0.674 \\
(0.000)\end{array}$ & $\begin{array}{c}0.576 \\
(0.003)\end{array}$ \\
\hline $\mathrm{X}_{6} \cdot \mathrm{WT}_{6}$ & - & $\begin{array}{c}0.967 \\
(0.000)\end{array}$ & $\begin{array}{c}0.836 \\
(0.000)\end{array}$ & $\begin{array}{c}0.847 \\
(0.000)\end{array}$ & $\begin{array}{c}0.851 \\
(0.000)\end{array}$ & $\begin{array}{c}0.744 \\
(0.000)\end{array}$ & $\begin{array}{c}0.818 \\
(0.000)\end{array}$ & $\begin{array}{c}0.821 \\
(0.000)\end{array}$ & $\begin{array}{c}0.697 \\
(0.000)\end{array}$ & $\begin{array}{c}0.670 \\
(0.000)\end{array}$ \\
\hline $\mathrm{X}_{2} \cdot \mathrm{WT}_{6}$ & $\begin{array}{c}0.575 \\
(0.003)\end{array}$ & $\begin{array}{c}0.564 \\
(0.003)\end{array}$ & $\begin{array}{c}-0.019 \\
(0.926)\end{array}$ & $\begin{array}{c}0.585 \\
(0.002)\end{array}$ & $\begin{array}{c}0.236 \\
(0.256)\end{array}$ & $\begin{array}{l}0.0453 \\
(0.830)\end{array}$ & $\begin{array}{c}0.256 \\
(0.217)\end{array}$ & $\begin{array}{c}0.021 \\
(0.922)\end{array}$ & $\begin{array}{c}-0.424 \\
(0.034)\end{array}$ & $\begin{array}{c}0.199 \\
(0.339)\end{array}$ \\
\hline $\mathrm{X}_{4} \cdot \mathrm{WT}_{6}$ & $\begin{array}{c}0.894 \\
(0.000)\end{array}$ & $\begin{array}{c}0.928 \\
(0.000)\end{array}$ & $\begin{array}{c}0.726 \\
(0.000)\end{array}$ & $\begin{array}{c}0.853 \\
(0.000)\end{array}$ & $\begin{array}{c}0.759 \\
(0.000)\end{array}$ & $\begin{array}{c}0.671 \\
(0.000)\end{array}$ & $\begin{array}{c}0.653 \\
(0.000)\end{array}$ & $\begin{array}{c}0.542 \\
(0.005)\end{array}$ & $\begin{array}{c}0.495 \\
(0.011)\end{array}$ & $\begin{array}{c}0.311 \\
(0.130)\end{array}$ \\
\hline $\mathrm{X}_{6} \cdot \mathrm{WT}_{6}$ & - & $\begin{array}{c}0.914 \\
(0.000)\end{array}$ & $\begin{array}{c}0.807 \\
(0.000)\end{array}$ & $\begin{array}{c}0.889 \\
(0.000)\end{array}$ & $\begin{array}{c}0.797 \\
(0.000)\end{array}$ & $\begin{array}{c}0.765 \\
(0.000)\end{array}$ & $\begin{array}{c}0.739 \\
(0.000)\end{array}$ & $\begin{array}{c}0.414 \\
(0.039)\end{array}$ & $\begin{array}{c}0.755 \\
(0.000)\end{array}$ & $\begin{array}{c}0.726 \\
(0.000)\end{array}$ \\
\hline $\mathrm{X}_{6} \cdot \mathrm{WT}_{6}$ & - & $\begin{array}{c}0.950 \\
(0.000)\end{array}$ & $\begin{array}{c}0.761 \\
(0.000)\end{array}$ & $\begin{array}{c}0.828 \\
(0.000)\end{array}$ & $\begin{array}{c}0.527 \\
(0.006)\end{array}$ & $\begin{array}{c}0.756 \\
(0.000)\end{array}$ & $\begin{array}{c}0.521 \\
(0.007)\end{array}$ & $\begin{array}{c}0.628 \\
(0.000)\end{array}$ & $\begin{array}{c}0.862 \\
(0.000)\end{array}$ & $\begin{array}{c}0.658 \\
(0.000)\end{array}$ \\
\hline \multicolumn{11}{|c|}{ Pooled data } \\
\hline $\mathrm{X}_{2} \cdot \mathrm{WT}_{6}$ & $\begin{array}{c}0.579 \\
(0.000)\end{array}$ & $\begin{array}{c}0.581 \\
(0.000)\end{array}$ & $\begin{array}{c}0.115 \\
(0.325)\end{array}$ & $\begin{array}{c}0.521 \\
(0.000)\end{array}$ & $\begin{array}{c}0.259 \\
(0.025)\end{array}$ & $\begin{array}{c}0.112 \\
(0.338)\end{array}$ & $\begin{array}{c}0.371 \\
(0.001)\end{array}$ & $\begin{array}{c}0.164 \\
(0.160)\end{array}$ & $\begin{array}{c}-0.016 \\
(0.886)\end{array}$ & $\begin{array}{c}0.186 \\
(0.110)\end{array}$ \\
\hline $\mathrm{X}_{4} \cdot \mathrm{WT}_{6}$ & $\begin{array}{c}0.918 \\
(0.000)\end{array}$ & $\begin{array}{c}0.930 \\
(0.000)\end{array}$ & $\begin{array}{c}0.664 \\
(0.000)\end{array}$ & $\begin{array}{c}0.727 \\
(0.000)\end{array}$ & $\begin{array}{c}0.748 \\
(0.000)\end{array}$ & $\begin{array}{c}0.609 \\
(0.000)\end{array}$ & $\begin{array}{c}0.660 \\
(0.000)\end{array}$ & $\begin{array}{c}0.605 \\
(0.000)\end{array}$ & $\begin{array}{c}0.541 \\
(0.000)\end{array}$ & $\begin{array}{c}0.404 \\
(0.000)\end{array}$ \\
\hline $\mathrm{X}_{6} \cdot \mathrm{WT}_{6}$ & - & $\begin{array}{c}0.941 \\
(0.000)\end{array}$ & $\begin{array}{c}0.788 \\
(0.000)\end{array}$ & $\begin{array}{c}0.847 \\
(0.000)\end{array}$ & $\begin{array}{c}0.707 \\
(0.000)\end{array}$ & $\begin{array}{c}0.751 \\
(0.000)\end{array}$ & $\begin{array}{c}0.714 \\
(0.000)\end{array}$ & $\begin{array}{c}0.618 \\
(0.000)\end{array}$ & $\begin{array}{c}0.755 \\
(0.000)\end{array}$ & $\begin{array}{c}0.679 \\
(0.000)\end{array}$ \\
\hline
\end{tabular}

$\mathrm{X}_{2} \cdot \mathrm{WT}_{6}=$ Correlation of traits of $2^{\text {nd }}$ week with $6^{\text {th }}$ week body weight; $\mathrm{X}_{4} \cdot \mathrm{WT}_{6}=$ Correlation of traits of $4^{\text {th }}$ week with $6^{\text {th }}$ week body weight; $\mathrm{X}_{6} \cdot \mathrm{WT}_{6}=$ Correlation of traits of $6^{\text {th }}$ week with $6^{\text {th }}$ week body weight. Figures in parenthesis indicate ' $P$ ' value. 
Table.3 Backward elimination regression model for prediction of $6^{\text {th }}$ week body weight from variables recorded at $2^{\text {nd }}$ week of age

\begin{tabular}{|c|c|c|c|c|}
\hline $\begin{array}{l}\text { Intercept with predictors } \\
\text { in final model }\end{array}$ & $\begin{array}{l}\text { Regression } \\
\text { coefficients }\end{array}$ & $\begin{array}{c}' P ' \text { - Value of } \\
\text { Regression coefficient }\end{array}$ & $\mathbf{R}^{2}(\%)$ & 'P' - Value of model \\
\hline \multicolumn{5}{|l|}{ Yellow } \\
\hline Constant & -21.571 & 0.407 & \multirow[t]{2}{*}{25.3} & \multirow[t]{2}{*}{0.010} \\
\hline Wing Length & 218.012 & 0.010 & & \\
\hline \multicolumn{5}{|l|}{ Black } \\
\hline Constant & 192.906 & 0.000 & \multirow[t]{4}{*}{59.6} & \multirow[t]{4}{*}{0.000} \\
\hline Body weight & 0.173 & 0.001 & & \\
\hline Breast Girth & -9.452 & 0.001 & & \\
\hline Thigh Length & -7.073 & 0.057 & & \\
\hline \multicolumn{5}{|l|}{ Stripped } \\
\hline Constant & 82.477 & 0.023 & \multirow[t]{3}{*}{36.0} & \multirow[t]{3}{*}{0.007} \\
\hline Body weight & 0.287 & 0.002 & & \\
\hline Breast Girth & -8.143 & 0.018 & & \\
\hline Constant & 1972.644 & 0.000 & 41.0 & 0.000 \\
\hline \multicolumn{5}{|l|}{ Pooled } \\
\hline Growth & 69.61 & 0.000 & & \\
\hline Breast Girth & -110.39 & 0.004 & & \\
\hline
\end{tabular}

$\mathrm{R}^{2}=$ Coefficient of determination of regression model 
Though certain conformation traits (shank, toe, thigh and wing length and breast girth) showed significant variation among the different plumage groups of CaribroDhanraja chickens, the results were not consistent. The FCR of Caribro- Dhanraja chickens observed in this study is more or less comparable with Anonymous (2003) and Bhonsle (2009), but higher than Tomar et al., (2011). Moreover, annual report of ICAR cited wide variation of FCR (1.65-2.15) at different centers (Anonymous, 2003).

\section{Correlation of growth traits with market weight}

Correlation of different growth traits at $2^{\text {nd }}, 4^{\text {th }}$ and $6^{\text {th }}$ week with $6^{\text {th }}$ week body weight is depicted in Table 2. Growth traits at $2^{\text {nd }}$ week and above age were positively and significantly correlated with live weight at $6^{\text {th }}$ week in all the three groups (yellow, black and stripped) as well as in pooled data; however, the degree of association differed among the groups. On the other hand, association of growth traits at $2^{\text {nd }}$ week with $6^{\text {th }}$ week varied among the different plumage groups. In yellow plumage group, the body weight, daily gain, weight: length ${ }^{2}$, shank length, breast length and thigh length at $2^{\text {nd }}$ week was positively associated with body weight at $6^{\text {th }}$ week. In black plumage group, body weight, daily gain and weight: length ${ }^{2}$ of $2^{\text {nd }}$ week was positively associated with $6^{\text {th }}$ week weight, but the association between thigh length of $2^{\text {nd }}$ week and body weight of $6^{\text {th }}$ week was negative. Similar to yellow and black plumage, body weight, daily gain and weight: length ${ }^{2}$ at $2^{\text {nd }}$ week was positively associated with $6^{\text {th }}$ week weight in stripped plumage group. In pooled data, there was positive and significant association of $2^{\text {nd }}$ week body weight, daily gain, weight: length ${ }^{2}$, shank length and breast length with $6^{\text {th }}$ week body weight. Previous studies reported positive association of growth traits with body weight at $6^{\text {th }}$ or $7^{\text {th }}$ week of age in Caribro- Dhanraja broiler chicken carried out by different authors at different parts of India (Singh, 2008; Singh and Jilani, 2008; Bhonsle, 2009; Tomar et al., 2011) and similar results were observed in this study. The degree of association of growth traits at $2^{\text {nd }}, 4^{\text {th }}$ and $6^{\text {th }}$ week with body weight at $6^{\text {th }}$ week increases with increase in age which is inconsonance with Singh (2008). Significant variation of correlation coefficient values among different plumage groups may be due to in consistent effect of plumage colour on growth. Positive association of early growth traits with market weight indicates that market weight could be predicted from early growth traits in Caribro- Dhanraja broiler chicken.

\section{Prediction of market weight}

The outputs of final backward elimination regression model for prediction of $6^{\text {th }}$ week body weight based on growth traits at $2^{\text {nd }}$ week are presented in Table 3. The regression model revealed higher accuracy $\left(\mathrm{R}^{2}=59.6 \%\right.$, $P<0.001)$ in black plumage group and the predictor variables were body weight, breast girth and thigh length. However, in yellow and stripped plumage groups the $\mathrm{R}^{2}$ values for prediction of $6^{\text {th }}$ week body weight using variables at $2^{\text {nd }}$ week were very small $\left(\mathrm{R}^{2}=\right.$ $25.3 \%, P=0.01$ and $\mathrm{R}^{2}=36.0 \%, P=0.007$, respectively). Moreover, regression analysis of pooled data resulted $\mathrm{R}^{2}$ value $41 \%$ and predictor variables were daily growth and breast girth. The results indicated marked effect of plumage colour on prediction of $6^{\text {th }}$ week body weight using variables at $2^{\text {nd }}$ week might be attributed to variation of different traits among the different plumage groups. Previous studies used different linear conformation traits for prediction of live weight and observed comparatively higher value of coefficient of determination of regression model (Latshaw and Bishop, 2001; 
Amao et al., 2011; Sanda et al., 2014). The predicted and explanatory variables of same age were regressed by the previous authors (Latshaw and Bishop, 2001; Amao et al., 2011; Sanda et al., 2014), but in this study $6^{\text {th }}$ week body weight was predicted using variables at $2^{\text {nd }}$ week. This may be reason of lower value of coefficient of determination of regression in Caribro- Dhanraja broiler chicken. Further, the linear traits for the final model also varied among the studies. This may be the reason of lower value of coefficient of determination of regression model in Caribro- Dhanraja broiler chicken. The results of this study indicated inconsistent effect of plumage on growth traits in CaribroDhanraja coloured broilers. Growth traits showed positive association with live body weight. Sixth week body weight could be predicted with higher accuracy from variables recorded at $2^{\text {nd }}$ week of age in black plumage group and the predictor variables were body weight, breast girth and thigh length. However, there was very less accuracy for prediction of $6^{\text {th }}$ week weight based on variable at $2^{\text {nd }}$ week for other plumage groups (yellow and stripped) and even for pooled data.

\section{Acknowledgement}

Authors are highly thankful to the Director of Research, Junagadh Agricultural University, Junagadh for providing all research facilities. Authors are also thankful to the technical and non-technical staffs of Poultry unit of Instructional Livestock Farm Complex, Veterinary College, Junagadh who helped during the research work.

\section{References}

Amao, S.R., Ojedapo, L.O., Oyewumi, S.O. and Ameen, S.A. 2011. Estimation of body weight from linear body measurements in Wadi Ross meat type chicken in derived savannah zone of Nigeria. Proceedings of the 16th Annual Conference of the Animal Science Association of Nigeria, Anyigba, Nigeria. pp. 9-12.

Anonymous. 2003. DARE/ICAR- annual report, 2002-2003. Indian Council of Agricultural Research, New Delhi.

Anonymous. 2017. Annual report, 2016-17. AICRP on Poultry Breeding and Poultry Seed Project. ICAR - Directorate of Poultry Research, Rajendranagar, Hyderabad.

Azahan, E.A.E. 1994. The red and black-red native chickens of Malaysia. MARDI Res. J., 22: 73-78.

Bett, H.K., Peters, K.J. and Bokelmann, W. 2011. Hedonic price analysis to guide in breeding and production of Indigenous chicken in Kenya. Livest. Res. Rural Dev, 23(6), http: //www.lrrd.org/ 1rrd23/6/bett23142.htm.

Bhonsle, D. 2009. Studies on growth, carcass characteristics and meat quality traits in improved varieties of chicken. Ph.D. Thesis, Indira Gandhi Krishi Vishwavidyalaya, Raipur (C.G.).

Chatterjee, S., Hadi, A.S. and Price, B. 2000. Regression analysis by example. John Willey \& Sons, Inc., New York.

Jiang, R.S. and Yang, N. 2007. Effect of dayold body weight on subsequent growth, carcass performances and levels of growth-related hormones in quality meat-type chicken. Archiv fur Geflugelkunde, 71(2): S93-96.

Latshaw, J.D. and Bishop, B.L. 2001. Estimating body weight and body composition of chickens by using noninvasive measurements. Poultry Sci., 80: 868-873.

Molenaar, R., Reijrink, I.A.M., Meijerhof, R. and Brand, H.V.D. 2008. Relationship between hatchling length and weight on later productive performance in broilers. World's Poultry Sci. J., 64(4): 599-604. 
Mukhtar, N., Khan, S.H. and Anjum, M.S. 2013. Hatchling length is a potential chick quality parameter in meat type chickens. World's Poultry Sci. J., 69(4): 889-896.

Padhi, M.K. 2016. Importance of indigenous breeds of chicken for rural economy and their improvements for higher production performance. Scientifica, 2016, http://dx.doi.org/ 10.1155/2016/ 2604685.

Patbandha, T.K., Garg, D.D., Marandi, S., Vaghamashi, D.G., Patil, S.S. and Savsani, H.H. 2017. Effect of chick weight and morphometric traits on growth performance of coloured broiler chicken. J. Entomol. Zool. Stud., 5(6): 1278-1281.

Rizzi, C. 2017. Plumage colour in Padovana chicken breed: growth performance and carcass quality. Italian J. Anim. Sci., DOI: 10.1080/1828051X.2017.1413598

Rizzi, C., Contiero, B. and Cassandro, M. 2013. Growth patterns of Italian local chicken populations. Poult Sci., 92: 2226-2235.

Sanda, A.J., Olowofeso, O., Adeleke, M.A., Oso, A.O., Durosaro, S.O. and Sanda, M.O. 2014. Heritability and repeatability estimates of some measurable traits in meat type chickens reared for ten weeks in Abeokuta, Nigeria. Int. Schol. Sci. Res. Innov., 8(7): 782-785.

Sauerbrei, W., Royston, P. and Binder, H. 2007. Selection of important variables and determination of functional form for continuous predictors in multivariable model building. Stat. Med., 26: 5512-5528.

Singh, C.B. 2008. Inheritance of growth and confirmation traits in CARI Dhanraja broiler strain. Indian J. Poultry Sci., 43(2): 243-244.

Singh, C.B. and Jilani, M.H. 2008. Inheritance of six-week body weight, shank length, keel bone length and breast angle in broiler chickens. Panthnagar J. Res., 6(2): 279-280.

Tomar, S., Saxena, B.K., Sachdev, A.K. and Ramgopal. 2011. Productivity evaluation of commercial "CaribroDhanraja" in different conditions. Bhartiya Krishi Anusandhan Patrika, 26(1 \& 2): 48-52.

Willemsen, H., Everaert, N., Witters, A., Smit, L.D., Debonne, M., Verschuere, F., Garain, P., Berckmans, D., Decuypere, E. and Bruggeman, V. 2008. Critical assessment of chick quality measurements as an indicator of post-hatch performance. Poultry Sci., 87(11): 2358-2366.

Wolanski, N.J., Renema, R.A., Robinson, F.E., Carney, V.L. and Fancher, B.I. 2006. Relationship between chick conformation and quality measures with early growth traits in males of eight selected pure or commercial broiler breeder strains. Poultry Sci., 85(8): 1490-1497.

Yang, N. and Jiang, R.S. 2005. Recent advances in breeding for quality chickens. World's Poultry Sci. J., 61: 373-381.

\section{How to cite this article:}

Patbandha, T.K., D.D. Garg, D.G. Vaghamashi, S. Marandi, K. Ravikala, S.S. Patil and Dash, S.K. 2018. Prediction of Market Weight in Caribro-Dhanraja Broilers with Different Plumage Colour Using Growth Traits. Int.J.Curr.Microbiol.App.Sci. 7(09): 1147-1155. doi: https://doi.org/10.20546/ijcmas.2018.709.136 